\title{
Fait, fiction et assomption : Les puissances cognitives de l'imagination selon Meinong
}

Par AURÉLIEN ZINCQ

FNRS, Université de Liège

There is nothing either good or bad but thinking makes it so. Shakespeare, Hamlet, II, 1.

There are more things in heaven and earth, Horatio, Than are dreamt of in your philosophy. Shakespeare, Hamlet, $V$.

Résumé L'objectif de cette étude est d'étudier la théorie de l'assomption élaborée par Alexius Meinong sous l'angle d'une réflexion sur le caractère cognitif de l'imagination. Grâce à sa théorie de l'assomption, le philosophe autrichien a en effet offert une interprétation de l'imagination qui va pleinement dans le sens d'une reconnaissance du caractère cognitif de celleci. Il s'agira de voir comment Meinong réussit à saisir la singularité de cette activité intellectuelle, dont le mode propre de réalisation semble être le discours, et dont le type d'objets qu'elle appréhende consiste en des objets concrets inexistants. Pour mener à bien ce projet d'une mise en tension de l'assomption et de l'imagination, il faudra passer par une lecture sémantique de la théorie de l'objet.

\section{Introduction}

Le concept d'imagination recouvre au moins deux acceptions qui, sans s'exclure l'une l'autre, ne se réduisent cependant pas. D'un côté, on entend en général par ce terme la faculté de l'esprit humain à produire des images dites «mentales»: les images qui nous passent par la tête (la Phantasie), le 
souvenir, le rêve ou encore l'hallucination. D'un autre côté, l'imagination peut désigner la capacité d'invention et de création propre à l'esprit humain. À ce pouvoir de création est indéniablement reconnue une dimension intellectuelle, qui s'exprime tant dans les sphères artistiques que scientifiques. Celui qui possède cette disposition à l'inventivité peut pénétrer au-delà du champ de ce qui est donné hic et nunc: il rend présent ce qui n'est pas donné effectivement. Jusqu'à un certain point, et dans les limites de leur domaine respectif, il est légitime de considérer que le physicien et le poète partagent cette même disposition à enrichir le réel de variantes imaginaires.

Dans cet article, je mettrai de côté l'étude du versant hylétique du concept d'imagination au profit d'une analyse de son versant cognitif. Je souhaiterais plus précisément me pencher sur la problématique des objets concrets inexistants. Cette problématique est à la fois d'ordre psychologique et ontologique. On peut la résumer en deux questions : (1) À quoi pense-t-on lorsque l'on pense à quelque chose qui n'existe pas ? (2) Et s'il est vrai que nous pouvons y penser - ce que l'on admettra assez facilement comment y pense-t-on en fait ? À chacune de ces questions correspond un aspect de l'acception intellectuelle de l'imagination. D'une part, on voit que l'imagination possède un pouvoir de conception: elle crée des objets. D'autre part, ce pouvoir de conception - qui fait donc d'elle un véritable acte intellectuel ou de cognition - est étroitement lié à au fait d'assumer (ce qui ne veut pas dire feindre) l'existence de ce qui aura été conçu.

La voie que j'emprunterai pour fournir une ébauche de réponse à ces deux questions passe par les travaux du philosophe autrichien Alexius Meinong (1853-1920). La problématique des objets inexistants - à entendre ici au sens large de tout ce qui n'est pas existant effectivement - a animé de part en part la philosophie élaborée par Meinong ${ }^{1}$. Il a d'ailleurs résumé celle-ci sous la forme d'un paradoxe devenu célèbre : " Il y a des objets à propos desquels on peut affirmer qu'il n'y en a pas $»^{2}$. Dans cette étude, je montrerai comment la tentative de solution que Meinong et ses étudiants ont donné à ce paradoxe - qui a pour nom la «théorie de l'objet»

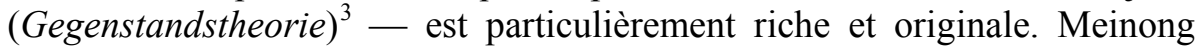

${ }^{1}$ Cf. F. Lavocat, Fait et fiction. Pour une frontière, Paris, Le Seuil, coll. « Poétique », 2016, p. 420.

2 A. Meinong, «La théorie de l'objet» (1904), trad. fr. J.-F. Courtine \& M. de Launay, dans Id., Théorie de l'objet et présentation personnelle, Paris, Librairie philosophique J. Vrin, coll. «Bibliothèque des Textes Philosophiques», 1999, § 3 , p. 73.

Cf. A. Meinong (dir.), Untersuchungen zur Gegenstandstheorie und Psychologie, Leipzig, J.A. Barth, 1904. 
réussit, du point de vue ontologique, à faire droit à la présence, au sein de notre monde, d'objets concrets inexistants, tout en offrant, du point de vue psychologique, une théorie qui rende compte de la façon dont nous nous rapportons à ces objets. Toutefois, la résolution du paradoxe des objets inexistants, telle qu'elle est proposée par Meinong, est seulement possible dès lors que l'on accepte de souscrire à une lecture sémantique de la théorie de l'objet. À mon avis, seule une telle interprétation sémantique permet à la théorie meinongienne de l'assomption de révéler l'essentiel des pouvoirs cognitifs de l'imagination.

\section{La théorie de l'objet et le préjugé en faveur de la réalité effective}

Dès ses débuts, la philosophie s'est interrogée sur le statut que l'on pouvait accorder à tous ces objets auxquels le discours se réfère, et qui pourtant ne semblent pas - parfois avec évidence - être le cas. Déjà Platon, dans Le Sophiste, se posait la question de savoir sur quoi portait un discours qui ne soit discours d'aucun sujet. Sa solution, comme on s'en souvient, consistait à affirmer que, si le discours ne portait sur rien, par exemple dans le cas où je dis «Théétète, avec lequel je parle, vole», alors il ne pouvait absolument pas être considéré comme un discours, étant entendu que, selon Platon, il est impossible qu'il y ait un discours qui soit un discours de rien $(263 c)$. En d'autres termes, tout discours authentique est nécessairement un discours sur quelque chose, et ce quelque chose doit être quelque chose d'étant, c'est-àdire d'existant ${ }^{1}$. Certes, on peut parler de ce qui n'est pas - ce que font allègrement les Sophistes - , mais parler du non-être revient toujours à se positionner par rapport à l'être. De la sorte, il n'y a pas de contraire à l'être : soit une chose existe, soit elle n'existe pas. Le non-être de quelque chose consiste en une opposition à l'être $(257 c)$. C'est à cette unique condition que Platon peut affirmer que le non-être participe à l'être $(260 d)^{2}$. Par ce geste

\footnotetext{
${ }^{1}$ Sur cette interprétation du Sophiste et la question de la solution intentionnaliste de la référence, $c f$. J. Benoist, Représentations sans objets: Aux origines de la phénoménologie et de la philosophie analytique, Paris, PUF, coll. "Épiméthée », 2001, p. 67-69.

2 Sur tout ceci, on consultera l' «Introduction» de N.L. Cordero à Platon, Le Sophiste, traduction et présentation de N.L. Cordero, Paris, Éditions Flammarion, coll. «GF Flammarion», 1993, p. 11-65. On pourra lire également les pages lumineuses de M. Dixsaut, Platon et la question de la pensée. Études platoniciennes I, Paris, Librairie philosophique J. Vrin, coll. « Bibliothèque d'Histoire de la Philosophie », 2000, p. 257 sq.
} 
de rapatriement du non-être dans le domaine de l'être - au sens où il est, par rapport à une Forme, ce qu'elle n'est pas - , Platon inaugure $^{1}$ ce qui allait devenir une tendance prégnante et constante de la métaphysique (ou, depuis le point de vue de Platon, de la philosophie) et que Meinong appellera, en 1904 , le « préjugé en faveur de la réalité effective » ${ }^{2}$. Le philosophe de Graz explique les raisons de ce préjugé par « l'intérêt particulièrement vif — qui nous est naturel - par nous porté à ce qui est effectivement réel », un intérêt qui, selon lui, " favorise cet excès qui consiste à traiter ce qui n'est pas effectivement réel comme un pur néant, plus précisément à le traiter comme quelque chose qui n'offrirait à la connaissance aucun angle d'attaque ou, du moins, aucun qui fut digne d'intérêt $»^{3}$. On peut toutefois s'étonner que Meinong qualifie la position platonicienne de "préjugé ». Comme il l'affirme lui-même, ne reflète-t-elle justement pas une conception intuitive et naturelle du discours? Pourquoi, dès lors, souhaiter la réformer ?

Que le discours doive porter sur quelque chose, c'est là sans aucun doute une nécessité, mais quant à avancer qu'il faille alors en convenir que ce quelque chose doive être un existant, il s'agit là d'une conséquence que Meinong - à rebours d'une bonne partie de l'histoire de la métaphysique refuse tout net, et contre laquelle il ne cessera de militer. Selon lui, en écartant l'idée qu'il soit possible de tenir un discours vrai sur ce qui n'est pas existant, on exclut de l'expérience - et on s'interdit ainsi de comprendre une large tranche de notre ontologie "domestique » et quotidienne - sans parler des entités dont traitent la physique et les sciences naturelles en général, voire l'histoire qui, enquêtant sur le passé, s'occupe d'événements ne participant plus, par définition, à l'être effectif. Or, qui souhaiterait soustraire de l'ensemble des objets avec lesquels nous commerçons, que ce soit de manière banale ou à l'aide de constructions théoriques complexes, tout ce dont nous n'avons pas d'expérience sensible? Une telle philosophie pourrait à bon droit être taxée - si, à nouveau, on n'a pas peur des paradoxes - d'abstraite et d'illusoire. Se préoccuper authentiquement et intégralement de l'ensemble des objets d'une expérience possible, c'est là une tâche certes ardue et exigeante, mais à laquelle toute philosophie qui prétend rendre compte de ce que cela signifie, pour l'homme, de « faire expérience », doit s'attacher. Cette tâche de parvenir à faire droit à la diversité des expériences possibles et à ce qu'elles peuvent recéler comme part de vérité - au sens où cela veut dire quelque chose de tenir pour vrai que Phileas

\footnotetext{
${ }^{1}$ Et assurément, en fait, Parménide avant lui.

${ }^{2}$ A. Meinong, « La théorie de l'objet » (1904), trad. fr., § 2, p. 67.

${ }^{3}$ Ibid., $\S 2$, p. 68.
} 
Fogg a fait le tour du monde en 80 jours — est précisément celle à laquelle la théorie de l'objet a pour but de s'atteler.

Dans cette perspective, on comprend mieux pourquoi la sentence selon laquelle « il y a des objets à propos desquels on peut affirmer qu'il n'y en a pas ${ }^{1}$ nous apparaît d'emblée comme un paradoxe : elle reflète cette tendance - le préjugé — de la métaphysique à ne donner de valeur qu'à la seule expérience sensible, au fait brut de la réalité, écartant la diversité des objets de connaissance et négligeant l'étude des différents niveaux ou couches d'être qui «stratifient» notre expérience quotidienne. Le paradoxe prend alors la forme d'un pari : réussir à le neutraliser, cela revient à faire droit, positivement et depuis notre monde quotidien, à ce que les deux questions posées plus haut qualifiaient d'objets « inexistants ». La théorie de l'objet, dans sa prétention à embrasser la totalité des objets d'une expérience possible, s'écarte ainsi dès le début de toute ambition systématique, et par là tendanciellement superficielle; elle est intégralement une "philosophie par le bas $»^{2}$.

Toutefois, avant que nous nous demandions ce qu'il faut entendre par cet élargissement du concept d'objet, il peut être utile d'indiquer brièvement les raisons pour lesquelles Meinong en vient à réclamer un tel élargissement de la catégorie fondamentale de l'ontologie - et par conséquent son dépassement. Qu'est-ce qui motive Meinong à prendre à rebrousse-poil toute l'histoire de la métaphysique et à lutter en faveur de l'inexistant ?

\footnotetext{
${ }^{1}$ Ibid., § 3, p. 73.

2 A. Meinong, " Présentation personnelle » (1920), trad. fr. J.-F. Courtine \& M. de Launay, dans Id., Théorie de l'objet et présentation personnelle, op. cit., p. 164. Bien sûr, « il est tout à fait possible de lire la théorie de l'objet comme un catalogue, dans lequel on trouverait énumérés, les uns à côté des autres, les différents genres d'objectivité » (J. Benoist, « La logique et l'épistémologie. Meinong et les niveaux de l'objectivité ?», Philopsis (revue électronique, sans numéro), 2010), mais considérer un tel catalogue — si, du reste, il s'agit bien de cela - comme un « système », cela serait en totale contradiction avec l'esprit et la lettre de la philosophie de Meinong et de ses étudiants : (1) la philosophie de Meinong est une philosophie radicalement empiriste, sans cesse remise en chantier, notamment par le partage des tâches ; (2) ce partage des tâches signifie concrètement la distribution du travail de recherche entre Meinong et ses étudiants, ce qui implique le dialogue, la mise en commun des résultats, la confrontation, etc., comme il y va dans une authentique et dynamique équipe de recherche; (3) cela serait aussi nier l'ambition de Meinong et de ses étudiants de se confronter à l'expérience : les catégories d'objets sont ancrées dans l'expérience, elles ne sont pas «flottantes » au-dessus de celle-ci.
}

\section{5}


Cette décision d'interroger «l'objet en tant que tel " s'enracine dans une question classique de psychologie philosophique, à savoir celle de la définition des actes mentaux. Comme cela est bien connu, Franz Brentano, dont Meinong fut l'élève à Vienne, définit la classe des actes psychiques par leur rapport à un objet : chaque processus psychique est intentionnel, c'est-àdire qu'il possède cette propriété intrinsèque d'être orienté vers quelque chose $^{1}$. Or, la question émerge rapidement de savoir ce qu'il en est de penser à un objet contradictoire, impossible, idéal, abstrait, fictif, etc. ${ }^{2}$ Comment un acte (bien réel) peut-il viser ce qui n'existe pas ? Chaque étudiant de Brentano (Carl Stumpf, Anton Marty, Alexius Meinong, Edmund Husserl pour ne citer que les plus célèbres), et Brentano lui-même, aura à répondre de cette question. Parmi les brentaniens, la position particulière de Meinong sera d'affirmer la possibilité d'une science capable d'un « traitement rigoureux de l'objet en tant que tel et dans sa généralité ${ }^{3}$. En d'autres mots, Meinong réaffirme, à la suite de Brentano, le caractère intentionnel de tout acte mental — le fait qu'il soit dirigé vers un objet - , tout en soutenant la thèse d'une indépendance radicale de l'objet visé par rapport à l'acte qui le vise. Il lui faut alors attester l'autonomie de l'objet eu égard à l'acte qui le vise - et de quelle autonomie quand il s'agit d'un objet fictif ou contradictoire - , et de ce rapport lui-même, qui est loin d'être évident. Nous retrouvons par là nos deux questions initiales.

\footnotetext{
${ }^{1}$ «Ce qui caractérise tout phénomène psychique, c'est ce que les Scolastiques du Moyen Âge ont appelé l'inexistence intentionnelle (ou encore mentale) d'un objet et ce que nous pourrions appeler nous-mêmes - en usant d'expressions qui n'excluent pas toute équivoque verbale - la relation à un contenu, la direction vers un objet (sans qu'il faille entendre par là une réalité (Realität) ou objectivité (Gegenständlichkeit) immanente. Tout phénomène psychique contient en soi quelque chose à titre d'objet (Objekt), mais chacun le contient à sa façon. Dans la représentation, c'est quelque chose qui est représenté, dans le jugement quelque chose qui est admis ou rejeté, dans l'amour quelque chose qui est aimé, dans la haine quelque chose qui est haï, dans le désir quelque chose qui est désiré et ainsi de suite » (F. Brentano, Psychologie du point de vue empirique, trad. fr. M. de Gandillac révisée par J.-F. Courtine, Paris, Librairie philosophique J. Vrin, coll. «Bibliothèque des Textes Philosophiques », 2008, p. 101-102).

${ }^{2}$ On peut renvoyer, sur cette question des « représentations sans objet» (au sens des représentations dirigées vers un objet «inexistant») à J. Benoist, Représentations sans objets: Aux origines de la phénoménologie et de la philosophie analytique, op. cit.

${ }^{3}$ A. Meinong, « La théorie de l'objet » (1904), trad. fr., § 1, p. 67.
} 


\section{Dépasser le donné sensible : vers l'idéalité ?}

Pourquoi la métaphysique ne pourrait-elle pas être cette science de l'objet dans sa généralité ? En effet, se demande Meinong, "si l'on songe à quel point la métaphysique a toujours eu pour intention d'intégrer au domaine de ses réflexions le plus proche comme le plus lointain, le plus grand comme le plus petit, il pourrait sembler en tout cas étrange qu'elle ne puisse assumer la tâche qu'on vient d'évoquer ${ }^{1}$, à savoir celle de parvenir à une science de l'objet en tant que tel et dans sa généralité, sans exclure a priori ce qui relève de l'inexistence. La réponse est pour Meinong fort simple : la métaphysique a sans doute «affaire à la totalité de ce qui existe, mais la totalité de ce qui existe - en y incluant ce qui a existé et ce qui va exister — est infiniment restreinte par rapport à la totalité des objets de connaissance $»^{2}$.

On objectera à Meinong qu'il est sans doute excessif de considérer que le domaine de recherche de la métaphysique a d'emblée exclu ce qui ne relevait pas de la réalité effective. La métaphysique ne s'est-elle pas aussi intéressée à ce qui se trouve au-delà de la réalité effective ? Meinong a répondu à cette objection :

Que l'« ontologie », la « doctrine des catégories », etc. n'aient jamais cessé d'être imputées, plus ou moins unanimement, à la compétence de la métaphysique - et que cela ait parfois pu également faire droit à des intérêts qui allaient au-delà des frontières de la réalité effective - témoigne seulement de la légitimité et du caractère impérieux de ces mêmes intérêts, mais ne laisse aucune place, pour autant que je puisse le constater, à un doute quant au fait que l'intention fondamentale de toute métaphysique n'a cependant jamais cessé de viser l'appréhension du «monde » au sens propre, au sens naturel, c'est-à-dire le monde de la réalité effective, même lorsque cette appréhension semblait déboucher sur ceci que ce qui devait être appréhendé ne pouvait aucunement prétendre à la qualification de réalité effective ${ }^{3}$.

Parmi les objets dont la métaphysique n'aurait pas tenu compte, qu'elle aurait considéré comme un "pur néant», Meinong recense en premier lieu les objets mathématiques (nombres, ensembles, figures géométriques), qui constituent un réseau d'objets auxquels on peut ajouter les relations telles que l'identité, la différence, le rapport, la ressemblance, l'analogie, etc. Tous ces objets — que Meinong qualifie d'« idéaux » pour insister sur leur irréductibi-

${ }^{1}$ Ibid., $\S 2$, p. 68 .

${ }^{2}$ Idem.

${ }^{3}$ Ibid., $\S 11$, p. 101-102. 
lité à l'existence effective, « réelle » — possèdent le statut ontologique de la subsistance (Bestehen). Précisons ce que cela signifie, pour un objet, de subsister. Si l'on prend l'exemple de la différence entre deux objets réels, on admettra sans hésitation qu'elle possède une forme d'être (Dasein) — ou, tout simplement, qu'elle est -, bien que celle-ci ne puisse être envisagée sur le même mode que les deux objets réels sur lesquels elle est fondée : on dira, par suite, qu'elle subsiste. Qu'en est-il dans le cas des objets mathématiques? Subsistent-ils, eux aussi ?

Jamais, insiste Meinong, l'être dont s'occupent les mathématiques en tant que telles n'est existence [au sens d'existence effective; AZ] ; eu égard à cela, jamais elles ne franchissent les limites de ce qui est doté d'une subsistance: une ligne droite n'a pas plus d'existence qu'un angle droit, un polygone régulier ou un cercle [-à quoi il faudrait bien sûr ajouter les nombres; $\mathrm{AZ}]^{1}$.

Que la ligne droite, pas plus que l'angle droit, ne puisse franchir les limites de son domaine pour atteindre celui de l'existence, il s'agit là d'un acte de classification que l'on pourrait qualifier, dans un premier temps, d' ' ontologique » : Meinong veut tout simplement dire que ces entités mathématiques ne peuvent prétendre à l'existence parce que le domaine de ce qui existe ne s'étend pas, par définition, au-delà de ce qui est donné hic et nunc dans une expérience sensible et rien que dans une expérience sensible. Ce qui existe possède une place définie dans l'espace et le temps. On pourrait de la sorte renverser ce que dit Meinong au sujet des objets mathématiques et l'appliquer au domaine de l'effectif : l'être de la table en tant que telle est existence, elle ne pourra donc jamais franchir les limites de l'existence. La table possède une situation définie dans l'espace et le temps qui la cantonne au domaine de l'Existieren.

C'est ce rapport à la temporalité qui nous permettra de décrire de manière plus exacte ce qui distingue ce qui existe effectivement de ce qui subsiste. Si cette distinction est au départ, pour Meinong, des plus naturelles et évidentes, il précise cependant que ce qui subsiste ne peut être affecté par le cours du temps. Ce qui subsiste est atemporel (zeitlos) ${ }^{2}$. Les pommes qui sont devant moi peuvent toutes deux devenir brunes en pourrissant, il n'en reste pas moins vrai - car c'est le cas - qu'au moment où je les perçois, elles sont bien d'une couleur différente ; la différence entre le rouge et le vert des pommes, à l'instant $t$ où elles furent perçues, m'est bel et bien donnée ; on peut donc dire à son propos qu'elle est, mais sur un mode d'être différent

${ }^{1}$ Ibid., § 2, p. 70 .

${ }^{2}$ A. Meinong, Über Annahmen, Leipzig, J.A. Barth, 1910², § 11, p. 64. 
de celui des objets perçus - et qui n'en est pas moins vrai ou le cas. Je peux par exemple saisir avec évidence que la différence de nuance de couleur entre ces deux pommes n'est pas la même qu'entre deux autres pommes. Bien sûr, il n'y a pas d'expérience sensible qui me présente directement ces différences - au sens où je pourrais l'appréhender en et pour elle-même mais il est tout à fait correct de dire que la différence est, qu'elle insiste depuis mon expérience sensible, ou encore qu'elle se manifeste à moi en tant que telle. La différence ne pourrit pas ; elle n'est pas affectée par le passage du temps; elle subsiste en dehors de tout cadre spatio-temporel, et c'est précisément parce qu'elle subsiste de la sorte qu'elle peut m'être donnée comme objet d'expérience.

\section{Par-delà l'être et le non-être}

Des questions relatives à l'extension du domaine de l'objet (Gegenstand) ne peuvent manquer de surgir. L'ensemble des objets de connaissance - au sens où, pour Meinong, tout objet est objet de connaissance car en droit connaissable, ce qui ne signifie rien de plus que le fait qu'il puisse être-donné (Gegebenheit) - est-il toutefois restreint aux relations, nombres, figures géométriques, etc. ? La subsistance épuise-t-elle l'ensemble de ce qu' «il y a » à côté de l'existence ? La totalité de l'existant et du subsistant constitue-telle la totalité des objets de connaissance ? Tout ce qui m'est donné doit-il inévitablement relever de ces deux domaines d'objets?

On peut d'emblée répondre par la négative à ces questions: non, l'ensemble de ce qu'il y a ne se réduit pas à ces deux catégories de l'existence effective (Existieren) et de la subsistance (Bestehen). Il existe, soutient Meinong, un savoir de la non-réalité, un savoir de l'absurde, des impossibilia (carré rond, montagne d'or, perpetuum mobile, etc.), des objets fictifs, etc. En d'autres mots, il faut aussi réserver un statut à ces étranges objets : pour affirmer que l'on ne trouve nulle montagne d'or sur Terre, n'est-on pas obligé de porter un jugement sur la montagne d'or? L'objet «montagne d'or » ne doit-il pas m'être donné d'une façon ou d'une autre ? Or, si un tel objet n'est reconnu comme appartenant ni au domaine de l'existant ni à celui du subsistant, quel statut lui réserver? N'ai-je pas la représentation d'une montagne d'or avant de reconnaître que l'on n'en trouve guère sur notre planète? Pour formuler cette question dans les termes ad hoc: que la représentation d'une montagne d'or soit sans objet existant ou subsistant, cela implique-t-il qu'elle soit pour autant sans objet? 
Par-delà les niveaux de ces deux types d'objets que nous pouvons nous représenter, il existe, «si l'on peut s'exprimer ainsi » précise Meinong ${ }^{1}$, une sorte de troisième niveau: celui de l'objet pur (reiner Gegenstand). Il ne s'agit pas là, à proprement parler, d'un niveau ontologique mais, beaucoup plus simplement, de tout ce qui, comme objet, peut être donné dans la représentation - indépendamment, il faut y insister, de toute question relative à son statut ontologique. Ce troisième domaine d'objets n'en est pas, en ce sens, authentiquement un - c'est d'ailleurs pourquoi Meinong parle d'Außersein : l'objet pur se trouve «jenseits von Sein und Nichtsein $»^{2}$. Dans un article qui lui est consacré, Dale Jacquette résume parfaitement la théorie meinongienne de l'Außersein :

La doctrine meinongienne de l'Außersein de l'objet pur postule un domaine sémantique d'absolue neutralité ontique. L'Außersein est littéralement au-delà de l'être. [...] L'Außersein n'est pas une sous-catégorie spécifique de l'ontologie, et l'Außersein n'est pas une sorte spéciale d'être, mais rentre entièrement en-dehors de l'ontologie des entités existantes et subsistantes, des objets qui possèdent l'être. [...] L'Außersein est en lui-même le domaine sémantique de tous les objets visés [all intented objects], quoiqu'il en soit de leur statut ontique, qui ne leur attribue pas un statut ontique ou quasi-ontique spécifique, qui ne serait pas celui de tous les autres objets ${ }^{3}$.

En bref, ajoute un peu plus loin Dale Jacquette :

L'Außersein de l'objet pur constitue le domaine sémantique de tous les objets compris uniquement en tant qu'objets, constitués exclusivement dans leur Sosein [être-tel] par leurs propriétés nucléaires, sans tenir compte de leur statut ontique $^{4}$.

\footnotetext{
${ }^{1}$ A. Meinong, « La théorie de l'objet » (1904), trad. fr., § 4, p. 75.

${ }^{2}$ Ibid., $\S 4$, p. 76. Il y a cependant certains passages de son œuvre où Meinong fait comme si l'Außersein constituait un troisième domaine d'objets mais, dans l'ensemble, c'est l'interprétation non ontologique qui semble la plus conforme à la pensée de Meinong.

${ }^{3}$ D. Jacquette, «Außersein of the Pure Object», dans L. Albertazzi, D. Jacquette \& R. Poli (éd.), The School of Alexius Meinong, Aldershot, Ashgate, coll. «Western Philosophy Series », 2001, p. 373, p. 383.
}

${ }^{4}$ Ibid., p. 383. 
Je ne reviendrai pas ici sur le bien-fondé de cette lecture sémantique de la théorie de l'objet ${ }^{1}$, qui a l'avantage d'éviter la plupart des problèmes qu'une lecture radicalement ontologique - qui, à suivre l'interprétation de Dale Jacquette, constituerait une contradictio in adjecto - ne manquerait pas de $\operatorname{poser}^{2}$. Ce qui m'importe pour le moment est de mettre en lumière le fait que Meinong reconnaît non pas l'existence (sous quelque forme que ce soit), mais bien plutôt la "présence sémantique $»^{3}$ de «tous les objets potentiels de la pensée $»^{4}$. En somme, il peut nous venir à l'esprit une infinité d'objets constitués des propriétés les plus diverses (mêmes contradictoires), sans que jamais l'on s'inquiète de l'existence, de la non-existence, de la possibilité, de la nécessité, etc., de ces objets ${ }^{5}$. Ces dernières propriétés, pour reprendre la métaphore utilisée par Meinong, sont dites extranucléaires ; elles ne relèvent donc pas du « noyau » des propriétés constitutives de l'objet qui me vient à la pensée. On prendra pour exemple le cas où un enfant qui, s'initiant à la géométrie euclidienne, pourrait avoir l'idée d'associer les propriétés de différentes figures géométriques pour former celle d'un carré rond, ou encore celui où, laissant vagabonder mon imagination, je me représente une créature fantastique dont le corps serait celui d'un lion et la tête celle d'un homme.

\footnotetext{
${ }^{1}$ L'article de Dale Jacquette (art. cit.) revient sur les différentes étapes qui ont conduit Meinong à élaborer sa théorie de l'Außersein de l'objet pur précisément dans un tel horizon sémantique. Pour une défense de cette lecture sémantique (et non pas, par conséquent, ontologique), on lira avec profit A. Sierszulka, Meinong on Meaning and Truth, Frankfurt, Ontos Verlag, coll. «Phenomenology and Mind», 2005. Pour une discussion des thèses défendues dans l'ouvrage d'A. Sierszulka, on lira la recension de V. Raspa dans Grazer Philosophische Studien, 76 (2008), p. 250-254.

${ }^{2}$ En bref, l'absence de reconnaissance du caractère sémantique des objets postulés dans l'Außersein meinongien impliquerait qu'il faille alors reconnaître à des objets tels que la montagne d'or et la machine à mouvement perpétuel une existence a mini$m a$, c'est-à-dire toujours une forme d'existence (mais qui ne serait pas celle de l'existence effective ni de la subsistance). On a bien du mal à imaginer où serait situé un tel domaine d'objets - étant entendu, comme Dale Jacquette le souligne, que l'Außersein comprend tous les objets possibles.

${ }^{3}$ D. Jacquette, art. cit., p. 373. C'est moi qui souligne.

${ }^{4}$ Ibid., p. 384.

${ }^{5}$ Sur les propriétés nucléaires et extranucléaires, $c f$. J. N. Findlay, Meinong's Theory of Objects and Values, Oxford, Clarendon Press, $1963^{2}$, p. 102-112 ; D. Jacquette, « Nuclear and Extranuclear Properties », dans L. Albertazzi, D. Jacquette \& R. Poli (éd.), The School of Alexius Meinong, op. cit., p. 397-426; F. Nef, « La théorie modale de Meinong », dans J.-P. Cometti \& K. Mulligan (dir.), La philosophie autrichienne de Bolzano à Musil: Histoire et actualité, Paris, Librairie philosophique J. Vrin, coll. «Problèmes \& Controverses », 2001, p. 81-99.
} 
Dans les deux cas, celui qui se représente ces objets ne se soucie nullement de savoir s'ils existent, dans ce monde-ci ou dans un autre; ces objets sont une pure création dont les éléments constitutifs ont été glanés dans le domaine des possibilités sémantiques - au sens large de tout ce à quoi il est permis de se référer sur les différents modes de la représentation - au sein duquel s'inscrit celui à qui ces objets étranges sont venus à la pensée.

Je laisse pour l'instant en suspens l'élucidation des raisons pour lesquelles Meinong élabore cette distinction entre propriétés nucléaires et extranucléaires de l'objet. Pour l'instant, il nous suffit de mentionner que cette attention portée aux propriétés nucléaires et extranucléaires - les secondes étant uniquement relatives au fait que quelque chose soit ou pas le cas, nécessaire, possible, probable, ou encore soit dit être selon les modes de l'existence effective ou de la subsistance - nous conduit directement au cœur de la théorie meinongienne du jugement. En effet, Meinong va transposer cette différence au cour de sa théorie des actes du discours. Cela ne nous fera cependant pas quitter les réflexions d'ordre « ontologique».

\section{La théorie des objectifs}

Pour Meinong, les actes discursifs sont des expériences psychiques qui nous confrontent à un type d'entités irréductibles aux objecta donnés dans la représentation : les objectifs (Objektive). Par exemple, quand j'affirme qu'il n'y a pas d'habitants sur Mars, ce à quoi je suis renvoyé dans mon expérience judicative n'a en quelque sorte aucun rapport avec Mars et ses habitants, mais tout simplement au fait qu'il n'y a pas d'habitants sur Mars. Dit autrement, « qu'il n'y a pas d'habitants sur Mars » est l'objet visé dans mon jugement. Les objectifs sont les objets immédiats du jugement — et, comme nous le verrons, de l'assomption -, par la médiation desquels la pensée discursive se réfère aux objets qui lui sont donnés dans la représentation ${ }^{1}$.

\subsection{Les objectifs comme objets d'ordre supérieur}

\footnotetext{
${ }^{1}$ Pour un exposé complet de la théorie meinongienne des objectifs, on consultera J. N. Findlay, Meinong's Theory of Objects and Values, op. cit., p. 59-101. Voir également la lecture de $\mathrm{H}$. Elie dans Le signifiable par complexe : La proposition et son objet. Grégoire de Rimini, Meinong, Russell (1937), Paris, Librairie philosophique J. Vrin, coll. « Bibliothèque d'Histoire de la Philosophie », 2000.
} 
Les objectifs peuvent être qualifiés d'« objets d'ordre supérieur » car ils sont ultimement des objets « fondés » dans des objecta, c'est-à-dire les objets qui nous sont donnés dans la représentation (Vorstellung) ${ }^{1}$. En ce sens, un objectif n'est pas un objet par soi seul; il doit être construit sur d'autres objets : ceux-ci peuvent être fournis soit via la représentation, dans le cas où ils sont des objecta, soit être eux-mêmes des objectifs (mais toujours également fondés sur des objecta). Par exemple, juger que la neige est blanche nécessite comme matériel cognitif l'objectum neige ${ }^{2}$. Mais dans la situation où j'affirme que la neige, qui est formée de milliers de cristaux, apparaît blanche à l'œil humain, le rapport à l'objectum n'est plus direct : les objectifs sont construits sur d'autres objectifs - ce qui n'empêche pas que l'on retrouve ultimement un objectum à leur fondement. Bien qu'ils soient d'un statut logique distinct ${ }^{3}$, les objectifs sont par conséquent des objets d'ordre supérieur de la même facture que les objets idéaux : ils ne peuvent subsister sans jamais avoir affaire à un objectum (qu'il soit existant ou subsistant) compris en eux à titre de moment ou de partie. Mais les objectifs possèdent-ils tous le mode d'être de la subsistance?

\subsection{Statut ontologique des objectifs}

En règle générale les objectifs, à l'instar des objets idéaux, ont pour mode d'être la subsistance ${ }^{4}$. Cependant, cela n'est exact que pour les objectifs

\footnotetext{
${ }^{1}$ On notera toutefois que les objectifs ne peuvent être envisagés comme appartenant à la même catégorie que les complexions, qui sont également des objets d'ordre supérieur (cf. A. Meinong, « Über Gegenstände höherer Ordnung und deren Verhältnis zur inneren Wahrnehmung » (1899), dans Id., Gesamtausgabe. Band II: Abhandlungen zur Erkenntnistheorie und Gegenstandtheorie, R. Haller \& R. Kindinger (Hrsg.), Graz, Akademische Druck- u. Verlagsanstalt, 1971).

2 Je reprends l'exemple de la neige et le terme de «matériel » à J. N. Findlay, Meinong's Theory of Objects and Values, op. cit., p. 71.

${ }^{3} C f$. la mise au point de J. Benoist, « La logique et l'épistémologie. Meinong et les niveaux de l'objectivité ? », art. cit.

${ }^{4}$ Je laisse ouverte, dans le cadre de cette étude, la question de savoir si les objets idéaux peuvent être donnés autrement que par un acte judicatif. Dans son article datant de 1899 «Sur les objets d'ordre supérieur et leur rapport à la perception interne », Meinong élabore la notion de Vorstellungsproduktion pour rendre compte du moyen par lequel nous réussissons à appréhender des objets complexes tels que les relations, les nombres, les qualités figurales, etc. Toutefois, dans la deuxième édition de son traité Über Annahmen, il indique que toute tentative pour produire une représentation complexe (c'est-à-dire un objet d'ordre supérieur idéal) est le produit
} 
vrais, que Meinong identifie avec ceux qui sont le cas. Un objectif faux ne possède pas le statut de la subsistance : il ne dénote aucun état de choses dans le monde - que cet état de choses soit positif ou négatif. Un objectif subsiste à la condition qu'il soit le cas, à savoir qu'il soit vrai. Meinong fait ici s'équivaloir l'être-vrai et le fait d'être le cas. Par conséquent, un objectif vrai fait plus que décrire un état de choses dans le monde, il est lui-même un tel état de choses. Qu'il y ait des êtres humains sur la Terre, cela est vrai parce qu'un tel état de choses est le cas : c'est un fait ; inversement, c'est parce que la présence d'êtres humains sur la Terre est un fait que l'objectif est vrai :

Selon Meinong, les seuls objectifs qui subsistent sont ceux qui sont des faits : la factualité est la modification spécifique de leur être [Being]; il y a une multitude infinie d'objectifs qui ne subsistent pas ${ }^{1}$.

Cette remarque de Findlay est infiniment précieuse : la factualité constitue une propriété de l'être de l'objectif, et non pas de l'être-tel; pour utiliser les catégories mentionnées précédemment, on dira que la factualité est une propriété extranucléaire de l'objectif. La possession d'une telle propriété implique la subsistance, le fait que l'objectif visé dans le jugement soit le cas et par conséquent vrai. La vérité et la fausseté, c'est-à-dire l'être ou pas le cas, sont des propriétés qui échoient aux objectifs. Quand une telle propriété échoit à l'objectif, elle entraîne une modification de son être qui le porte alors à épouser le mode de la subsistance.

Dans ces pages, je ne développerai pas dans le détail les implications de la théorie meinongienne de la vérité, qui semble à bien des égards plutôt déroutante. On remarquera simplement qu'en affirmant qu'il est le cas qu'un objet existe ou encore soit nécessaire, le plan sur lequel se situe l'objet de mon jugement est toujours celui de la subsistance. Quand je soutiens l'existence de la vie sur Terre, le fait qu'il y ait de la vie sur la Terre constitue un état de choses auquel il est reconnu la subsistance. À peu de choses près, cela revient à dire que la reconnaissance de l'existence implique la subsistance de ce qui est reconnu comme existant. De même, quand je certifie qu'une victoire de la droite aux prochaines élections est mathéma-

d'un travail discursif: l'acte de production va ainsi rapidement prendre la forme d'une activité de type judicatif, suggérant que ce qui relève de la subsistance en général ne peut nous être présenté que par l'intermédiaire d'un jugement ou d'une assomption ; le discours apparaît par là comme ce qui révèle l'idéalité au sein du monde « réel». Sur les objectifs comme états de choses dont l'appréhension est le produit d'un acte judicatif, $c f$. infra.

${ }^{1}$ J. N. Findlay, Meinong's Theory of Objects and Values, op. cit., p. 83. 
tiquement impossible, la vérité de mon énoncé rend factuel l'état de choses impossible qui y est décrit. Les diverses propriétés extranucléaires qui peuvent être associées à ce qui est jugé - et qui ne peuvent jamais l'être que par l'intermédiaire du discours - font de l'objet visé un état de choses dont la réalité vient en un sens se superposer à la réalité existante.

Dans cette perspective, ce n'est pas le jugement qui peut être dit vrai ou faux, pas plus qu'un objectum : la vérité et la fausseté sont des propriétés qui leur sont étrangères. Bien sûr, ainsi que le remarque Findlay, cela ne signifie pas qu'il y aurait des entités qui seraient vraies par nature :

Un fait est vrai en lui-même pour autant qu'il est l'objet d'un jugement. Il est possible, pour Meinong, de dire d'un fait qu'il est vrai parce que pour lui le mot « fait » n'est pas un simple nom mais implique une description. Les faits ne sont pas une ultime sorte d'entités, mais sont ces objectifs auxquels une ultime caractéristique appartient, la factualité ${ }^{1}$.

Comme on le voit, on se trouve ici à la frontière du logique, voire du sémantique $^{2}$, et de l'ontologique. L'objet du jugement est vrai pour autant qu'il est un fait ; mais il ne m'est cependant donné en tant que fait que pour autant qu'il est l'objet d'un jugement. La subsistance n'est pas accordée de droit à l'objectif : elle ne lui est octroyée précisément qu'en vertu d'un acte discursif. La notion d'objectif dépasse ainsi le discours où elle s'inscrit. Elle renvoie intimement à la possibilité, pour le discours, de complexifier notre expérience, de nous mettre en présence d'objets qui, s'ils sont certes fondés sur des objecta, ne s'y réduisent cependant pas. À cet égard, le cas des faits négatifs est particulièrement éclairant.

Si l'on parle d'un vote qui s'est déroulé sans perturbation, alors que la séance parlementaire qui l'avait précédé fut houleuse, ce dont il est question ici est un fait négatif. Toutefois, vu qu'il n'y a pas pour Meinong de représentation négative, mais que la négation fait directement basculer du côté de l'objectif parce qu'il s'agit d'affirmer que quelque chose n'est pas, on voit bien comment il revient à l'objectif de nous orienter vers un tel fait : c'est par l'intermédiaire de l'objectif qu'il est possible de se référer à ce qui ne peut être représenté - bien que ce fait, tout négatif qu'il soit, n'en constitue pas moins un événement, indépendant de sa prise en charge judicative. Qu'il n'y ait pas de perturbation lors du vote, c'est là un fait, mais un fait qui ne nous est accessible qu'à travers le jugement que l'on porte à son égard.

${ }^{1}$ Ibid., p. 90.

${ }^{2} \mathrm{~J}$. Benoist, Représentations sans objets : Aux origines de la phénoménologie et de la philosophie analytique, op. cit., p. 103. 
Pour Meinong, c'est l'objectif qui accomplit la visée authentique de ce qui est donné par le canal de la représentation. En d'autres termes, il n'est d'expérience « réelle » ou " complète » que de ce qui est embrigadé dans le discours ${ }^{1}$. Nous aurons l'occasion de discuter plus profondément ce versant de la théorie meinongienne des actes de discours quand nous aborderons, dans la suite de cet article, les perspectives ontologiques ouvertes par le traité de 1902 Sur les assomptions.

\subsection{Etre et être-tel}

En postulant une différence entre ce qui revient à l'être-tel de ce qui est jugé et à son être, Meinong établit par là même une distinction entre deux types d'objectifs : ceux qui concernent précisément l'être-tel et ceux qui concernent son être $^{2}$. Affirmer que Phèdre est la fille de Minos et de Pasiphaé, cela revient à viser un objectif relatif à l'être-tel ; par contre, affirmer que Phèdre existe, cela revient à juger de son être. Dans le premier cas, le jugement nous confronte à un objectif de l'être-tel (Soseinsobjektiv); dans le second, nous avons affaire à un objectif de l'être (Seinsobjektiv). Cette reconnaissance d'une disjonction entre les objectifs de l'être-tel et de l'être est ce que Meinong appelle, à la suite de son étudiant Ernst Mally, le principe de l'indépendance de l'être-tel par rapport à l'être ${ }^{3}$. Selon ce principe, ce qui est dit de quelque chose est affranchi de tout rapport à l'être de ce quelque

\footnotetext{
${ }^{1}$ A. Meinong, « Présentation personnelle » (1920), trad. fr., p. 143, p. 154. On consultera également R. Poli, "General Thesis of the Theory of Objects ", dans L. Albertazzi, D. Jacquette \& R. Poli (éd.), The School of Alexius Meinong, op. cit., p. 356 et J. N. Findlay, Meinong's Theory of Objects and Values, op. cit., p. 73.

${ }^{2}$ En passant, on notera comment Meinong prend à nouveau à contre-pied la tradition en théorie du jugement : alors qu'une bonne part de celle-ci identifiait le jugement sur l'être-tel avec celui sur l'être (comme si prédiquer impliquait automatiquement que ce sur quoi on a prédiqué possède une forme d'existence), le philosophe autrichien va découpler les jugements se rapportant à l'être de ceux se rapportant à l'êtretel. On retrouve là encore cette démarche empiriste si caractéristique de la pensée meinongienne : il est évident que lorsque j'affirme que le Père Noël passe par la cheminée pour apporter les cadeaux aux enfants, cela n'implique pas que je pose l'existence du Père Noël. On peut tout à fait énumérer les attributs d'un objet sans affirmer dans le même temps que cet objet participe à la réalité effective.

${ }^{3}$ E. Mally, «Untersuchungen zur Gegenstandstheorie des Messens », dans A. Meinong (dir.), Untersuchungen zur Gegenstandstheorie und Psychologie, op. cit., p. 126. Cf. K. Lambert, Meinong and the Principle of Independence, Cambridge, Cambridge UP, 1983.
} 
chose. Pour formuler ce principe plus simplement, ce n'est pas parce que j'ajoute des propriétés à un objet que je considère qu'il existe, subsiste, qu'il est possible ou encore qu'il est un fait. Les propriétés ajoutées à un objet par la pensée sont libres de toute position quant aux modalités ontologiques qui peuvent échoir à cet objet.

En dépit de cette distinction entre les objectifs de l'être et ceux de l'être-tel, il ne faudrait pas les considérer comme irréconciliables. Au contraire, il faut bien plutôt les envisager comme pouvant s'associer diversement l'un à l'autre. À nouveau, la perspective globale à l'intérieur de laquelle il faut saisir cette séparation entre les deux types d'objectifs est celle de l'inclusion de l'inférieur dans le supérieur: de la même façon que nous avons vu que les objectifs renvoyaient ultimement, et peu importe leur complexité, à des objecta, les objectifs de l'être « englobent» ceux qui sont relatifs à l'êtretel.

La mise en évidence de ce rapport d'inclusion nous permet de montrer comment la théorie de l'Außersein est étroitement connectée avec le principe d'indépendance. Il ne serait d'ailleurs pas erroné d'affirmer que la théorie de l'Außersein repose sur le principe d'indépendance ou, plus exactement, qu'ils se supportent mutuellement. Si, en effet, la validité du principe d'indépendance se manifeste de manière exemplaire dans les situations où l'on ne souhaite pas concéder à ce dont on parle une quelconque propriété relative à l'être (factualité, possibilité, nécessité, etc.), c'est aussi et avant tout parce que les matériaux qui composent ce sur quoi on juge - sans avoir d'intérêt pour son être - nous sont fournis par le domaine sémantique de l'Außersein. Pour le dire autrement, nos expériences discursives puisent dans l'Außersein comme dans ce fonds où se trouvent tous les objets potentiels de la pensée ${ }^{1}$. L'Außersein constitue de la sorte le domaine englobant de toute expérience discursive possible - et donc de toute expérience complète ; il est ce qui nous est littéralement donné à penser. Ainsi,

si quelqu'un juge, par exemple, qu' « un perpetuum mobile n'existe pas », alors il est tout à fait clair que l'objet dont l'existence [Dasein] est ici contestée doit posséder des propriétés, à savoir des propriétés caractéristiques, sans lesquelles la conviction de son inexistence ne pourrait avoir ni sens ni justification; posséder des propriétés signifie naturellement la même chose

${ }^{1}$ D. Jacquette, «Außersein of the Pure Object », art. cit., p. 384. 
que « être tel» [Sosein]. Ce dernier ne suppose cependant alors pas d'existence [Existenz], laquelle est justement, et avec raison, contestée ${ }^{1}$.

À ce stade, on ne voit pas encore très bien quel est le résultat escompté de cette capacité cognitive consistant à pouvoir affirmer que, en vertu de son être-tel, l'être-doré de la montagne d'or est similaire à celui du masque mortuaire d'Agamemnon ou qu'étant donné les propriétés contradictoires mais qui lui sont inhérentes - du perpetuum mobile, il est vrai d'affirmer qu'une machine qui prétendrait produire un mouvement perpétuel ne pourrait pas exister. Avant d'indiquer les raisons pour lesquelles le principe d'indépendance et la théorie de l'Außersein s'articulent l'un à l'autre, je procéderai à un bref récapitulatif de ce qui a été avancé à propos de la théorie meinongienne des objectifs.

\subsection{Les objectifs : que retenir?}

De ce parcours à travers la théorie meinongienne des objectifs - à la fois dans ses ramifications logiques et ontologiques - , deux éléments essentiels sont à retenir pour la suite de notre étude.

(1) En premier lieu, on insistera sur ceci que, pour Meinong, il doit toujours y avoir un objet « positivement» donné au jugement — qui lui est donc logiquement antérieur ${ }^{2}$ - même dans le cas où il s'agit d'affirmer qu'un tel objet ne participe pas à l'être, par exemple à cause de ses propriétés contradictoires - e.g. le carré rond n'est qu'une possibilité sémantique, il n'acquerra jamais la subsistance ${ }^{3}$. Avant que l'on affirme quoi que ce soit à propos de quelque chose, ce qui peut être dit émane du schème sémantique à partir duquel le locuteur porte un jugement. Ce que Meinong appelle l'Außersein désigne l'arrière-plan sémantique depuis lequel il peut y avoir discours. L'Außersein pourvoit à la pensée ce dont elle a besoin pour fonc-

\footnotetext{
${ }^{1}$ A. Meinong, Über Annahmen, op. cit., § 12, p. 79. Cf. également A. Meinong, « La théorie de l'objet » (1904), trad. fr., § 4, p. 74.

${ }^{2}$ A. Meinong, « Présentation personnelle » (1920), trad. fr., p. 167.

${ }^{3}$ Pour revenir à Platon, on voit donc que cela a un sens - parce qu'un objet est toujours déjà prédonné, donc logiquement antérieur (cf. supra) - de poser qu'il puisse y avoir un discours de rien : le cercle carré m'est donné comme objet sémantique, dont je peux en toute vérité affirmer qu'il possède telles et telles propriétés, bien qu'un jugement d'existence indique qu'un tel objet ne puisse être le cas. $C f$. Platon, Le Sophiste, op. cit., p. 196 (263 c).
} 
tionner ou, mieux encore, ce dont, depuis le schème sémantique d'où elle agit, il lui est permis de faire l'expérience.

(2) Tous ces objets qui, pour filer la métaphore, «se proposent» à la pensée sont au final embrigadés dans un discours qui va progressivement les constituer en objets d'une expérience possible. Comme on l'a vu, les objectifs qui sont le cas complexifient notre monde : ils sont comme une strate d'objets qui vient se superposer aux existants. L'exemple du fait négatif est à cet égard particulièrement frappant: il n'est pas possible de s'en faire une représentation, nous n'en réalisons l'expérience que par l'entrée en scène d'une activité judicative. C'est ce deuxième point ici mis en évidence qui va nous permettre d'apprécier l'utilité de la théorie meinongienne du jugement pour la problématique des objets dits « inexistants ». On entrevoit en effet la portée pratique du rôle que Meinong fait jouer aux actes discursifs.

\section{Pragmatique de l'assomption}

Dès le début de cet article, j'ai insisté sur des exemples concrets où sont mobilisés des objets dits " inexistants », par exemple des objets provenant de la fiction. D'ailleurs, en partant de notre expérience commune, j'ai même affirmé que l'on pouvait tout à fait donner une valeur de vérité aux jugements à propos de tels objets - il est vrai que Phileas Fogg a fait le tour du monde en 80 jours, tout aussi vrai que Phèdre est la fille de Minos et de Pasiphaé. Que l'on puisse dire quelque chose de vrai à propos de ce qui n'existe pas, c'est là une position que la tradition logique s'est bien souvent abstenue de défendre car elle semble impliquer que ce sur quoi porte le jugement participe effectivement à l'existence. Or, dès que le concept d'existence est réduit à l'existence effective - au sens de ce qui peut exercer un effet -, Meinong a montré que l'on arrive rapidement à ce paradoxe qu'il y a des objets qui n'existent pas. Nous avons vu que Meinong tâche de résorber ce paradoxe en travaillant à deux niveaux : d'une part, il affirme que ce paradoxe est seulement apparent car l'existence effective n'est pas le seul mode de l'être - on ne peut parler stricto sensu de l'existence d'un objet fictif puisque, par nature, il n'est pas un objet relevant de la catégorie de l'existence au sens d'existence effective; d'autre part Meinong, grâce à sa théorie de l'objet pur et au principe d'indépendance, va montrer qu'il est possible de porter un jugement sur l'être-tel d'un objet sans que cela n'engage ontologiquement celui qui porte ce jugement.

Mais la liberté qu'à peu près tout ce qui nous vient à l'esprit puisse également devenir l'objet d'un jugement vrai signifie-t-elle pour autant que, 
depuis notre «horizon sémantique ${ }^{1}$, nous accordions du crédit à tout ce dont nous avons l'idée? Plus fondamentalement encore, par quels moyens est-il possible de rapatrier un objet provenant de l'Außersein et, lui reconnaissant alors le droit à la subsistance, de lui concéder la factualité ? Que Flaubert puisse avoir l'idée d'un personnage tel que Madame Bovary, cela ne suffit pas encore à lui accorder suffisamment d'être pour qu'il soit vrai d'affirmer qu'elle est née Rouault ou qu'elle tombe amoureuse de Léon Dupuis. Pour tenter de comprendre le processus par lequel un objet simplement pensé devient un complexe qui puisse être le cas, il est nécessaire de faire un pas au-delà de la théorie de l'Außersein et du principe d'indépendance. Ce pas supplémentaire nous mène au cœur de la philosophie meinongienne : la théorie des assomptions (Annahme).

\subsection{Jugement et assomption}

Qu'est-ce qu'une assomption ? D'un point de vue strictement doctrinaire, une assomption est un jugement auquel il manque la conviction (Überzeugung $)^{1}$. D'après Meinong, le jugement est un acte qui possède deux composantes : d'une part, la position de l'état de choses jugé (qui peut aussi être négatif) et, d'autre part, la conviction de ce en quoi on juge. Ainsi, quand j'affirme que Ganymède est un satellite de Jupiter, l'objet direct (i.e. l'objectif) de mon jugement est que Ganymède est un satellite de Jupiter, auquel j'accorde donc foi. En dissociant ces deux moments du jugement, Meinong va mettre au jour une nouvelle classe d'actes psychiques, intermédiaire au jugement et à la représentation. Pour reprendre notre exemple, on peut imaginer la situation d'un jeu où, devant citer le nom des «lunes galiléennes » de Jupiter, j'avance, mais sans y croire, les noms des quatre grands satellites joviens - dont Ganymède. L'objectif de mon acte discursif est donc bel et bien identique à celui d'un jugement ; ce qui distingue cependant les deux actes est que, dans le cas de l'assomption, je ne crois en quelque sorte pas à ce que je dis être le cas ; plus précisément : la valeur de vérité est suspendue - même si, dans l'exemple, l'objectif visé est le cas. En bref, à l'objectif de l'assomption, qui vaut donc aussi pour le jugement, peut être attribuée la propriété de la factualité - à la condition que l'objectif supposé soit le cas - , bien que cela en fait importe peu car, à celui qui suppose, il manque précisément la conviction de ce qu'il avance : on se trouve donc en-

${ }^{1}$ R. Brisart, «La théorie des assomptions chez le jeune Husserl », Philosophiques, 36/2, 2009, p. 400. 
deçà de la valeur de vérité. Quel est l'avantage de pouvoir se dispenser du moment de la conviction dans le jugement?

Quand je suggère que, grâce à l'assomption, on se trouve dans l'acte discursif comme en-deçà de la valeur de vérité, il ne faut pas entendre cette proposition dans le sens où l'objectif visé par l'assomption ne pourrait être le cas ou encore parce que la vérité de ce qui est avancé serait purement et simplement suspendue ${ }^{2}$. Il ne faut pas considérer l'acte d'assomption uniquement du point de vue de la possibilité de pouvoir affirmer à peu près tout de tout, sans qu'il n'y ait jamais à contester ce qui est avancé. Au contraire, il faut bien plutôt y voir la possibilité de pouvoir poser ses propres normes de référence ; l'assomption se trouve en-deçà de la valeur de vérité parce qu'elle la pose. Quand on prolonge le geste meinongien de suspension du moment de la conviction, on constate alors que l'assomption peut être vue comme un discours qui établit ses propres normes de vérité. Alors que, dans le jugement, j'ai la conviction que ce que j'affirme est le cas, la suspension de la conviction ouvre, au sein du discours, un espace permettant l'élaboration d'un monde d'objets - ou, plus exactement d'états de choses — dont la factualité n'est pas le terminus ad quem. Là où le jugement emploie le mode de l'indicatif, l'assomption lui préfère le conditionnel. Reprenons notre exemple précédent.

Si j'affirme que Ganymède est une lune de Jupiter, j'ai alors la conviction que la propriété d'être une lune de Jupiter appartient intrinsèquement à l'être-tel de Ganymède. Quand je suggère que Ganymède pourrait être une lune de Jupiter, la propriété ainsi postulée pourrait vraiment relever de l'êtretel de Ganymède, mais c'est là un point sur lequel je n'ai aucune conviction. Dans les deux cas, à l'objectif appréhendé peut être associée la propriété de la factualité : que je sois convaincu de ce que j'affirme, ou pas, il est le cas que Ganymède soit une lune de Jupiter. C'est donc à la factualité que veut en venir directement le jugement: il veut qu'à ce qu'il pose soit d'emblée reconnu le fait d'être le cas. L'assomption est moins pressée: Ganymède pourrait désigner une lune de Jupiter, mais ne serait-ce pas aussi le nom du fils de Tros et de Callirrhoé ?

Cette prégnance du conditionnel dans l'acte d'assomption nous ramène au fait que la distinction entre les objectifs de l'être et de l'être-tel, si elle

${ }^{1}$ Cf. A. Meinong, Über Annahmen, op. cit., § 1, p. 1-8.

${ }^{2}$ Sur la distinction entre jugement hypothétique et assomption, $c f$. R. de Calan, "L'objectif de l'Objektiv: De l'objet du jugement à la théorie de l'objet », dans J. Benoist (éd.), Propositions et états de choses : Entre être et sens, Paris, Librairie philosophique J. Vrin, coll. « Problèmes \& Controverses », 2006, p. 109-110. 
permet effectivement d'accorder une plus grande amplitude quant à ce qui est exprimé au sein des actes discursifs, se voit néanmoins toujours soumise à l'éventualité d'un télescopage de l'objectif de l'être sur l'objectif de l'êtretel. Entre les deux types d'objectifs, on est donc toujours déjà dans un rapport de proximité et de distance; tantôt ils s'évitent, tantôt ils fusionnent. Au cœur de ce rapport complexe, l'assomption fait jaillir le conditionnel dans la théorie des actes discursifs : elle pose un domaine où les choses pourraient être telles ou telles. C'est dans cette optique qu'il y a, selon Meinong, un principe de liberté complète de l'assomption ${ }^{1}$ : non pas à entendre comme la possibilité de postuler des objets sans limites, mais bien plutôt à comprendre comme le choix pour le traçage de limites au sein desquelles les états de choses postulés peuvent venir s'intégrer à notre monde et le complexifier.

\subsection{Le sens du possible}

Meinong n'élabore pas une typologie des différents actes d'assomption, mais en recense les principaux domaines d'activité. Voici la liste, telle qu'elle est établie par Meinong dans le chapitre IV de Über Annahmen, des situations les plus évidentes où, selon lui, se manifestent les assomptions :

Les assomptions «explicites»-qui sont le plus souvent introduites par des phrases telles que « J'assume que... », « Je suppose que... », «Imagine que...» - sont présentes dans tous les contextes où l'on désire insister sur la présence de la démarche assomptive. Une telle démarche est typique des mathématiques. On prendra l'exemple de la démonstration par l'absurde, qui consiste à établir la validité d'une thèse en la supposant fausse en commençant la démonstration ou, plus généralement, les cas où il s'agit de dériver d'un ensemble d'axiomes une série de théorèmes (les géométries non euclidiennes fonctionnent sans postuler l'axiome des parallèles et en assumant un axiome contraire). Bien sûr, ce type d'assomptions explicites se retrouve fréquemment dans la vie quotidienne, où il n'est pas rare de réfléchir sous un mode hypothétique, que ce soit pour soi-même ou lors d'une

${ }^{1}$ A. Meinong, Über Annahmen, op. cit., § 60, p. 346. 
discussion ${ }^{1}$. On notera que le raisonnement par assomption constitue l'un des moteurs du dialogue socratique ${ }^{2}$.

Les assomptions sont également très présentes dans les sciences (et pas uniquement en mathématiques). Selon la façon dont elles sont utilisées par les scientifiques, on parlera alors plutôt d'hypothèses, voire de fictions. Meinong n'établit cependant pas une classification très précise dans ce chapitre IV à ce sujet ${ }^{3}$.

Dans le domaine moral, l'assomption explicite peut jouer un rôle déterminant. Il n'est pas rare de se retrouver dans ce genre de situation où nous disons « Mets-toi à ma place, et réfléchis à la façon dont tu aurais alors agis $\gg{ }^{4}$. Ce type de raisonnement est aussi sans doute l'une des bases du raisonnement de l'enquêteur ou du chercheur: non pas seulement la fabrication d'une hypothèse, mais cette forme d'introjection consistant à tenter d'adopter le point de vue d'autrui, c'est-à-dire de supposer qu'il aurait agi de telle ou telle façon étant donné un ensemble de facteurs définis.

Les assomptions sont très présentes dans les domaines du théâtre et de l'art. Les exemples sont nombreux. On peut agir en dissimulant son intention véritable; on peut prétendre être Hamlet, faire "comme si » ${ }^{5}$ Untel était vraiment Hamlet, et Hamlet un Prince de Danemark; on peut lire Madame Bovary comme un épisode réel d'une chronique provinciale française au $\mathrm{XIX}^{\mathrm{e}}$ siècle et, partant, anticiper la façon dont elle agit, se mettre à sa place, avoir de la peine pour elle, etc. ; on peut émettre des hypothèses quant à la signification d'un tableau abstrait.

Les jeux d'enfants sont pour Meinong un cas paradigmatique du champ d'application des assomptions. Dans ce cas, l'assomption est considé-

\footnotetext{
${ }^{1}$ Dans un processus de communication, je peux inférer une signification de ce que j'ai appréhendé comme complexe sonore, etc. $C f$. E. Dölling, «On Alexius Meinong's Theory of Signs », dans R. Poli (éd.), The Brentano Puzzle, Aldershot, Ashgate, coll. «Western Philosophy Series », 1998, p. 210-211.

${ }^{2}$ Je dois au Professeur Venanzio Raspa cette réflexion sur le concept d'assomption.

${ }^{3}$ X. de Donato-Rodríguez, « Meinong's Theory of Assumption and Its Relevance for Scientific Contexts », Meinong Studies, vol. 6, 2016, p. 141-173.

${ }^{4}$ A. Meinong, Über Annahmen, op. cit., § 15, p. 109.

${ }^{5}$ À peu près à la même époque que Meinong, Hans Vaihinger a rédigé, s'inspirant de Kant, sa Philosophie du comme si (1911). Il serait intéressant d'analyser les points de rencontre et de rupture entre la théorie des assomptions et la philosophie du « comme si ». Pour une introduction à la philosophie de Vaihinger, cf. C. Bouriau, Le "Comme si » : Kant, Vaihinger et le fictionalisme, Paris, Le Cerf, coll. « Passages », 2013. J'ai tenté une critique de cette approche dans le Bulletin d'analyse phénoménologique, IX/3, 2013 (Recensions 1), p. 9-15.
} 
rée comme un acte de «fantaisie» (Phantasie). Il mentionne le cas « d'illusion » où l'enfant fait semblant que la chaise sur laquelle il est assis est un cheval tirant un chariot "représenté » par la table : "Que la chaise qui a été harnachée à la table comme un cheval à un chariot est réellement considérée par l'enfant comme un cheval et que la table est vraiment considérée comme un chariot », voilà une situation courante où l'enfant en train de jouer se trouve engagé tout à la fois intellectuellement et imaginairement, sans que l'on puisse départager ces deux dimensions. Une telle situation ne fonctionne qu'à la condition de la présence d'un acte intellectuel de supposition : celuici soutient la «transformation » des différents objets (la table et la chaise) en d'autres objets (le cheval et le chariot). Ces objets sont précisément « fantasmés" au sens où l'illusion que ces objets sont bel et bien autres ne fonctionnent que si l'on suppose qu'ils le soient. Seul un acte de supposition à la base du contexte (l'enfant suppose que la chaise est un cheval) permet à celui-ci de devenir une activité ludique, et donc que les objets «en jeu » apparaissent à l'enfant tels qu'il se les imagine.

Meinong mentionne le mensonge comme acte d'assomption: «Le menteur ne croit pas lui-même à ce qu'il suppose être l'apparence de la croyance ${ }^{1}$. En d'autres mots, le menteur est celui qui pose un état de choses comme vrai, tout en sachant que la propriété de la factualité ne lui revient pas.

Meinong mentionne également les situations où un militaire participe à des manœuvres dans une académie, ou encore lorsqu'il s'agit de participer à un exercice d'alerte incendie, etc.

Dans toutes les situations qui viennent d'être présentées, on voit comment l'acte d'assomption permet l'élaboration de ce que l'on pourrait appeler un "contexte discursif» au sein duquel des potentialités vont émerger de l'expérience pour la complexifier, la rendre autre que ce qu'elle est. L'assomption, c'est l'expérience vécue d'un autre point de vue que ce qui se manifeste d'emblée et avec évidence. En elle, les choses sont vues non pas comme elles sont mais comme elles pourraient être. Pour reprendre la formule musilienne, l'assomption déploie « le sens du possible ${ }^{2}$.

\subsection{Assomption et imagination}

\footnotetext{
${ }^{1}$ A. Meinong, Über Annahmen, op. cit., § 17, p. 117.

${ }^{2}$ Cf. J.-P. Cometti, Musil philosophe. L'Utopie de l'essayisme, Paris, Le Seuil, coll. «Le Don des Langues», 2001, p. 75.
} 
Meinong considère l'assomption comme « une extension du concept d'imagination qui irait du registre intellectuel au registre émotionnel et, partant, s'étendrait jusqu'à toutes les classes fondamentales de l'expérience intime $»^{1}$. Cette extension du champ d'application de l'assomption ne nous étonnera pas au vu de ce qui a été avancé dans la section précédente. L'assomption peut avoir une fonction strictement intellectuelle, par exemple dans le domaine des mathématiques, mais aussi, plus généralement, quand elle désigne cette capacité de l'esprit humain à envisager des mondes possibles (qu'il s'agisse de la fiction ou de la science). En ce qui concerne le registre émotionnel, elle intervient quand il nous faut nous projeter dans toute sorte de mondes littéraires ou de fiction. Du côté de l'expérience intime, on se doute que la référence de Meinong au mensonge, à la dissimulation ou encore au jeu témoigne de l'incursion des actes assomptifs dans notre quotidien le plus propre.

Le concept d'assomption tel qu'il est élaboré par Meinong vient enrichir la conception courante de l'imagination, qui voit souvent en elle soit la production d'images mentales, soit une activité de création d'illusions et orientée vers la tromperie, la feintise et le faux-semblant. Dans le premier cas, on en reste à une version "psychologiste » de l'imagination ${ }^{2}$; dans le deuxième cas, on reste en-deçà de ses potentialités créatrices.

\footnotetext{
${ }^{1}$ A. Meinong, « Présentation personnelle » (1920), trad. fr., p. 124. On mentionnera que Meinong a écrit un article entièrement consacré aux représentations de la Phantasie (cf. A. Meinong, "Phantasievorstellung und Phantasie» (1889), dans Id., Gesamtausgabe. Band I: Abhandlungen Zur Psychologie, R. Haller \& R. Kindinger (Hrsg.), Graz, Akademische Druck- u. Verlagsanstalt, 1969). Cet article est écrit dans un esprit «brentanien » car Meinong, à l'époque, n'était pas encore parvenu à la théorie de l'objet.

${ }^{2}$ Je ne développerai pas ici une comparaison entre les actes d'imagination (au sens précis de l'occurrence d' « images mentales ») et d'assomption. Cf. A. Meinong, Über Annahmen, op. cit., §65, p. 375-384. On notera la proximité des deux affirmations de Meinong selon lesquelles, d'une part, l'assomption constitue un acte intermédiaire entre la représentation et le jugement et, d'autre part, qu'elle possède une affinité avec l'imagination, et ce que Kant avance, essentiellement dans la première édition de la Critique de la raison pure sur l'imagination transcendantale comme faculté intermédiaire entre la sensibilité et l'entendement. Bien entendu, le partage kantien entre sensibilité et entendement ne tient pas en registre brentanien (la classe des représentations ne se limite pas aux représentations sensibles... qui inclut également les représentations conceptuelles, qui sont dévolues, pour Kant, à l'entendement), mais la proximité est suffisamment grande pour être notée et, le cas échéant, devenir l'objet d'une étude plus approfondie. On pointera néanmoins que l'assomption meinongienne, en tant que radicalement discursive, n'a rien du carac-
} 
Dans la perspective défendue par Meinong, l'imagination est étudiée en tant que processus essentiellement discursif. L'invitation à imaginer « imagine que...» - dépasse la seule capacité à se représenter : elle ouvre un espace propre - et donc normé - qui unit à la fois des éléments pratiques et discursifs, par exemple dans le cas du jeu, où le jardin devient l'enceinte d'un château, un bâton une épée, etc. ${ }^{1}$ Il ne s'agit pas au final d'un simple faire « comme si », qui garde toujours par-devers lui l'assurance de la réalité vraie et évidente, mais il y va bien plutôt de l'ouverture à une réalité qui vient complexifier l'être-effectif. L'espace ainsi ouvert par l'assomption ne vient pas se superposer, étranger et donc toujours illusoire, à la réalité effective : il lui est au contraire coextensif, dans sa matérialité même, la compliquant, l'emmenant en quelque sorte au-delà d'elle-même mais toujours au titre de ce dont il y a authentiquement expérience. Par-là, le monde du jeu n'est pas un faux monde, un monde illusoire ou un arrière-monde : il possède ses règles propres, sa matérialité, ses objets particuliers.

Cette conception discursive de l'imagination ouvre sans doute à une interprétation inédite d'une "faculté» longtemps considérée comme une version dégradée de la perception et dont on a maintes fois soupçonné le potentiel créateur sans pouvoir toutefois dépasser la conception psychologiste et voir en quoi elle nous confrontait à des objets bien tangibles. En insistant à la fois sur la pluralité des usages de l'assomption et sur la grande fréquence avec laquelle nous l'intégrons diversement dans notre vie quotidienne, Meinong entreprend ce que l'on pourrait appeler une archéologie de l'imaginaire. Il montre comment l'imaginaire, sous la forme de la puissance discursive de l'assomption, participe à la dimension de l'agir.

\section{Assomptions et objets inexistants}

Quelles sont les conséquences de la théorie meinongienne de l'assomption pour la problématique des objets inexistants?

Comme nous l'avons vu, l'assomption constitue une forme de discours qui pose ses propres normes de vérité. Or en affirmant que, sous régime d'assomption, il y a élaboration des règles à partir desquelles il devient valable d'associer les objectifs de l'être à ceux de l'être-tel, Meinong pointe la finalité essentiellement «pratique» de l'assomption. Il « réhabilite»au

tère psychologisant de la faculté kantienne de l'imagination, qui reste encore prisonnière d'une vision «mentaliste» de l'imagination.

${ }^{1}$ A. Meinong, Über Annahmen, op. cit., § 16. 
sein du monde quotidien — au sens où il en montre la positivité — un ensemble d'objets que l'on aurait eu trop vite tendance à rejeter au-delà des limites du domaine où s'applique la valeur de vérité — ou, pour être plus précis, où l'on décide qu'elle s'y applique. En réalité, beaucoup de nos comportements et de nos énoncés ne sont valides - et cela n'infléchit en rien leur validité - qu'en régime d'assomption. Sous l'assomption de la « validité » du monde créé par Jules Verne, il est vrai d'affirmer que Phileas Fogg vit au 7, Savile-Raw à Londres et faux d'affirmer qu'il habite au 221b, Baker Street. Sous l'assomption du monde créé par Flaubert dans Madame Bovary, il est vrai - parce que c'est le cas - d'affirmer que l'épouse du Docteur Charles Bovary est née Rouault, et faux qu'elle tombe amoureuse du pharmacien Homais. La manière dont nous agissons ou nous parlons n'a bien souvent de sens - ou de valeur de vérité - que sous assomption. Cette puissance de l'assomption à poser les fondements de certains actes discursifs témoigne au final du caractère englobant de l'assomption eu égard au jugement. La suspension de la conviction et l'accès au conditionnel n'empêche pas de poser un jugement depuis le régime d'assomption.

Il est important d'insister sur la finalité pratique de l'assomption, qui permet d'intégrer théoriquement, comme objets d'une expérience possible, des objets que la philosophie n'a pas toujours réussi à intégrer au domaine de l'expérience. Il y va donc d'un élargissement de la catégorie de l'expérience. Le concept d'assomption, en nous permettant de nous mettre en situation " sous hypothèse », permet d'évoquer des objets qui ne sont pas présents hic et nunc. De la sorte, la liberté totale d'assomption, associée à la théorie de l'objet pur, permet de rendre compte du fonctionnement de la pensée aux prises avec la création, par exemple artistique, mais aussi mathématique, conceptuelle, etc. En d'autres termes, il y a là la possibilité de comprendre $d u$ point de vue psychologique, voire "psychologiste »", comment l'esprit vient à appréhender ou saisir des "pensées » et les met en forme au sein d'un discours cohérent ou encore de ce que cela fait que de se référer à un objet qui ne possède pas de statut défini. On peut ainsi tenter de saisir le « devenirmatériel » d'objets sémantiques qui prendront bel et bien forme au sein d'un discours déterminé.

\footnotetext{
${ }^{1}$ Pour utiliser l'expression de Tim Crane au sens où il l'emploie dans The Objects of Thought, Oxford, Oxford UP, 2013, p. 169.
} 


\section{Conclusion}

Je souhaiterais clore la réflexion menée dans cette étude en tâchant de reprendre les différentes idées qui y ont été avancées dans la perspective d'une interprétation globale de la philosophie meinongienne.

Je voudrais tout d'abord préciser que le point de vue exégétique à partir duquel fut appréhendée la théorie de l'objet s'oppose dans une large mesure à cette idée fort répandue - et, à mon avis, fausse - selon laquelle la théorie de l'objet pourrait être illustrée par la métaphore de la jungle. Je ne reviendrai pas ici dans le détail sur les différentes versions que cette métaphore a connues chez les commentateurs de Meinong. Je dirai simplement que, si l'on entend par là cette thèse que la théorie de l'objet constitue un vaste répertoire d'entités (des plus simples aux plus loufoques) auxquelles Meinong aurait reconnu une quelconque forme d'existence, alors on est loin d'avoir apprécié les subtilités du texte meinongien, mais on est aussi étranger que possible à l'esprit dans lequel la philosophie de Meinong fut élaborée. Meinong n'est pas, comme le pensait Ryle, «le plus grand multiplicateur d'entités de l'histoire de la philosophie ${ }^{1}$. En termes de niveaux d'objectivité, il reconnaît ceux de l'existence et de la subsistance; en ce qui concerne le deuxième mode, on ne trouvera pas cela trop extravagant: il est également reconnu par Husserl dans les Recherches logiques, et par bon nombre de logiciens.

Il faut être reconnaissant à une certaine orientation dans les approches sémantiques de la théorie de l'objet — je pense en particulier à Dale Jacquette et à Anna Sierszulka - d'avoir permis de sortir de cette ornière que fut malheureusement pour Meinong son interprétation radicalement ontologique. Toutefois, ces lectures sémantiques doivent elles-mêmes être considérées comme un pas en direction d'un autre type d'approche de la théorie de l'objet, peut-être encore plus en phase avec son esprit et sa lettre.

L'ambition de cette étude fut de prolonger le geste exégétique de ces approches sémantiques en élevant la théorie de l'objet au rang d'une " pragmatique ». Par-là, il s'agit de revenir aux exigences empiristes et descriptives qui furent celles de Meinong et de ses étudiants, et de montrer comment elles visent à rendre compte de nos "pratiques discursives », à accorder du crédit à nos usages sémantiques et à la façon dont, à travers ces pratiques et ces usages, nous élaborons un monde d'objets qui nous est propre. En ce sens,

${ }^{1}$ Cité dans D. F. Lindenfeld, The Transformation of Positivism. Alexius Meinong and European Thought (1880-1920), Berkeley-Los Angeles-London, University of California Press, 1980, p. 205. 
cette lecture "pragmatiste» de la théorie de l'objet — contrairement aux lectures ontologiques et, dans une moindre mesure, sémantiques - ne vise rien d'autre que la prise en charge de son ambition initiale.

C'est à mon avis dans le cadre de cette lecture d'abord sémantique et ensuite pragmatiste de la théorie de l'objet que les recherches de Meinong sur le concept d'assomption sont les plus fructueuses. Grâce au concept d'assomption, Meinong met en lumière un type d'acte psychique (qu'il situe entre la représentation et le jugement) dont on avait jusqu'alors peu compris la nature et, ce faisant, parvient à rendre compte des processus cognitifs régissant en quelque sorte le pouvoir de création intellectuelle souvent reconnu à l'imagination. Cette faculté, désormais sous le nom d'assomption, désigne un pouvoir cognitif de l'esprit à élaborer, par le biais du discours et c'est pourquoi une interprétation sémantique de la théorie de l'objet est nécessaire - des mondes possibles. On a vu l'ensemble des activités où Meinong aperçoit l'empreinte de l'assomption. Certes, il faudrait sans doute être plus détaillé, à l'avenir, sur cette multitude de situations les plus diverses, mais il n'en reste pas moins que, dans chaque cas, il y va de cette puissance de l'esprit à capter le possible, ce qui pourrait être dans ce qui est déjà.

\section{Bibliographie}

Benoist J., Représentations sans objets : Aux origines de la phénoménologie et de la philosophie analytique, Paris, PUF, coll. « Épiméthée », 2001.

Benoist J., «La logique et l'épistémologie. Meinong et les niveaux de l'objectivité ? », Philopsis (revue électronique, sans numéro), 2010.

Bouriau C., Le «Comme si »: Kant, Vaihinger et le fictionalisme, Paris, Le Cerf, coll. « Passages », 2013.

Brentano F., Psychologie du point de vue empirique, trad. fr. M. de Gandillac révisée par J.-F. Courtine, Paris, Librairie philosophique J. Vrin, coll. « Bibliothèque des Textes Philosophiques », 2008.

Brisart R., «La théorie des assomptions chez le jeune Husserl », Philosophiques, 36/2, 2009, p. 399-425.

Cometti J.-P., Musil philosophe. L'Utopie de l'essayisme, Paris, Le Seuil, coll. « Le Don des Langues », 2001.

Cordero N.L., «Introduction », dans Platon, Le Sophiste, traduction et présentation de N.L. Cordero, Paris, Éditions Flammarion, coll. «GF Flammarion», 1993, p. 11-65. 
Crane T., The Objects of Thought, Oxford, Oxford University Press, 2013.

De Calan R., «L'objectif de l'Objektiv: De l'objet du jugement à la théorie de l'objet », dans J. Benoist (éd.), Propositions et états de choses : Entre être et sens, Paris, Librairie philosophique J. Vrin, coll. « Problèmes \& Controverses », 2006, p. 87-118.

De Donato-Rodríguez X., « Meinong's Theory of Assumption and Its Relevance for Scientific Contexts », Meinong Studies, vol. 6, 2016, p. 141-173.

Dixsaut M., Platon et la question de la pensée. Études platoniciennes I, Paris, Librairie philosophique J. Vrin, coll. «Bibliothèque d'Histoire de la Philosophie », 2000.

Dölling E., « On Alexius Meinong's Theory of Signs », dans R. Poli (éd.), The Brentano Puzzle, Aldershot, Ashgate, coll. « Western Philosophy Series », 1998, p. 199-215.

Elie H., Le signifiable par complexe: La proposition et son objet. Grégoire de Rimini, Meinong, Russell (1937), Paris, Librairie philosophique J. Vrin, coll. « Bibliothèque d'Histoire de la Philosophie », 2000.

Findlay J.N., Meinong's Theory of Objects and Values, Oxford, Clarendon Press, $1963^{2}$.

Jacquette D., «Außersein of the Pure Object », dans L. Albertazzi, D. Jacquette \& R. Poli (éd.), The School of Alexius Meinong, Aldershot, Ashgate, coll. « Western Philosophy Series », 2001, p. 373-396.

Jacquette D., «Nuclear and Extranuclear Properties », dans L. Albertazzi, D. Jacquette \& R. Poli (éd.), The School of Alexius Meinong, Aldershot, Ashgate, coll. « Western Philosophy Series », 2001, p. 397-426.

Lambert K., Meinong and the Principle of Independence, Cambridge, Cambridge UP, 1983.

Lavocat F., Fait et fiction. Pour une frontière, Paris, Le Seuil, coll. « Poétique », 2016.

Lindenfeld D.F., The Transformation of Positivism. Alexius Meinong and European Thought (1880-1920), Berkeley-Los Angeles-London, University of California Press, 1980.

Mally E., «Untersuchungen zur Gegenstandstheorie des Messens », dans A. Meinong (dir.), Untersuchungen zur Gegenstandstheorie und Psychologie, Leipzig, J.A. Barth, 1904, p. 121-262.

Meinong A. (dir.), Untersuchungen zur Gegenstandstheorie und Psychologie, Leipzig, J.A. Barth, 1904.

Meinong A., Über Annahmen, Leipzig, J.A. Barth, $1910^{2}$.

Meinong A., "Phantasievorstellung und Phantasie » (1889), dans Id., Gesamtausgabe. Band I: Abhandlungen Zur Psychologie, R. Haller \& R. Kindinger (Hrsg.), Graz, Akademische Druck- u. Verlagsanstalt, 1969.

Meinong A., « Über Gegenstände höherer Ordnung und deren Verhältnis zur inneren Wahrnehmung » (1899), dans Id., Gesamtausgabe. Band II: Abhandlungen zur Erkenntnistheorie und Gegenstandtheorie, R. Haller \& R. Kindinger (Hrsg.), Graz, Akademische Druck- u. Verlagsanstalt, 1971. 
Meinong A., Théorie de l'objet et présentation personnelle, trad. fr. J.-F. Courtine \& M. de Launay, Paris, Librairie philosophique J. Vrin, coll. «Bibliothèque des Textes Philosophiques », 1999.

Nef F., «La théorie modale de Meinong », dans J.-P. Cometti \& K. Mulligan (dir.), La philosophie autrichienne de Bolzano à Musil : Histoire et actualité, Paris, Librairie philosophique J. Vrin, coll. «Problèmes \& Controverses », 2001, p. 8199.

Platon, Le Sophiste, traduction et présentation de N.L. Cordero, Paris, Éditions Flammarion, coll. « GF Flammarion », 1993.

Poli R., " General Thesis of the Theory of Objects », dans L. Albertazzi, D. Jacquette \& R. Poli (éd.), The School of Alexius Meinong, Aldershot, Ashgate, coll. «Western Philosophy Series », 2001, p. 347-372.

Sierszulka A., Meinong on Meaning and Truth, Frankfurt, Ontos Verlag, coll. «Phenomenology and Mind», 2005. 